\section{Letter: May Cooler Heads Prevail During a Pandemic: Stroke in COVID-19 Patients or COVID-19 in Stroke Patients?}

To the Editor:

Along with the novel coronavirus (COVID-19) pandemic comes a growing public fear of its severe complications and possible central nervous system involvement. ${ }^{1}$ Neurointerventionalists in COVID-19 prevalent regions raised an alarm toward an increased risk of acute large-vessel-occlusion stroke in COVID19 patients. It has been proposed that large-vessel stroke could be the presenting symptom of COVID-19 in younger patients. ${ }^{2}$ This has brought upon heightened fear and anxiety among younger adults. Yet, the scientific literature lacks evidence supporting a higher risk of stroke with COVID-19 in mildly symptomatic patients, or providing a causal link between COVID-19 and largevessel stroke in younger adults.

Systemic inflammation that may occur after severe COVID19 infection promotes a prothrombotic state that may predispose to thrombotic events, including stroke. However, stroke as the presenting complaint of COVID-19 patients with otherwise mild or no systemic disease raises concerns over COVID-19 being a bystander given its high prevalence in reporting regions. ${ }^{2}$ In a report of 5 patients younger than $50 \mathrm{yr}, 2$ of whom were underscored for having no stroke comorbidities, stroke was described as a presenting symptom of young COVID-19 patients. $^{2}$ This led to a robust news coverage to alarm young healthy adults of stroke secondary to COVID-19. We find this alarm unfounded. Stroke is less common, but not rare, in young adults. The findings presented remain anecdotal and lack the methodological and statistical rigor to claim that COVID-19 infection increases stroke risk in the youth or that stroke could be the presenting symptom of COVID-19. In fact, our Stroke Thrombectomy and Aneurysm Registry reports that $12 \%$ of 5000 patients undergoing thrombectomy are younger than $50 \mathrm{yr}^{3,4}$ Of young adults, $47 \%$ had no stroke comorbidity. Similarly, $8 \%$ of patients from the stroke thrombectomy trials were under 50 yr. $^{5}$ Therefore, anecdotal reports of small case series of stroke in younger adults with COVID-19 do not reach the threshold, to date, to raise public fear among young adults.
Although sounding early alarms during outbreaks may prevent delayed responses to novel threats, sufficient evidence is required to limit unnecessary burdens of the alarm itself on the healthcare system and public. Efforts to increase stroke awareness are always commended to avoid delay in patient presentation; however, in times of uncertainty and anxiety, it is critical to minimize misinformation that can have profound ramifications, even if it were well intended. Physicians are encouraged to seek sound medical evidence during care for patients and news appearances.

\section{Disclosures}

The authors have no personal, financial, or institutional interest in any of the drugs, materials, or devices described in this article.

\author{
Ali M. Alawieh, MD, PhD $\odot^{*}$ \\ Alejandro M. Spiotta, MD $\oplus^{\ddagger}$ \\ ${ }^{*}$ Department of Neurosurgery \\ Emory University School of Medicine \\ Atlanta, Georgia \\ *Department of Neurosurgery \\ Medical University of South Carolina \\ Charleston, South Carolina
}

\section{REFERENCES}

1. Mao L, Wang M, Chen S, et al. Neurological manifestations of hospitalized patients with COVID-19 in Wuhan, China: a retrospective case series study. JAMA Neurol. published online: April 10, 2020 (doi:10.1001/jamaneurol.2020.1127).

2. Oxley TJ, Mocco J, Majidi S, et al. Large-vessel stroke as a presenting feature of COVID-19 in the young. N Engl J Med. 2020;382(20):e60.

3. Alawieh A, Vargas J, Fargen KM, et al. Impact of procedure time on outcomes of thrombectomy for stroke. J Am Coll Cardiol. 2019;73(8):879-890.

4. Chalhoub RM, Alawieh AM, Anadani M, et al. Abstract 169: a comprehensive multicenter evaluation of the impact of age on stroke thrombectomy outcomesinsights from the STAR collaboration. Stroke. 2020;51:A169.

5. Goyal M, Menon BK, van Zwam WH, et al. Endovascular thrombectomy after large-vessel ischaemic stroke: a meta-analysis of individual patient data from five randomised trials. Lancet. 2016;387(10029):1723-1731.

Copyright (C) 2020 by the Congress of Neurological Surgeons

10.1093/neuros/nyaa283 